\title{
Test section of the wind tunnel IT for aeroelastic experiments with blade cascades
}

\author{
Václav Vlček ${ }^{1}$, Pavel Procházka ${ }^{1, *}$ \\ ${ }^{1}$ Institute of Thermomechanics, Czech Academy of Sciences, Czech Republic
}

\begin{abstract}
The article presents the way how the existing small vacuum aerodynamic tunnel IT (Institute of Thermomechanics) has been adapted for the measurement of the aeroelastic properties of the NACA 0015 airfoil and of the blade cascades composed of various types of blades with two degrees of freedom, pitch and common plunge. Attention was focused on the possibility of studying self-excited vibration at lower subsonic speeds. The modification of the test section is based on the knowledge gained during the study of self-excited airfoil oscillation.
\end{abstract}

\section{Introduction}

The problem of aeroelasticity of profile cascades is mentioned in the literature rarely $[1,2,3,4]$ and the information about the construction of an aerodynamic tunnel for the aeroelasticity of blade cascades with twoor more degrees of freedom has not been found in the studied literature. Also, in IT laboratories, aeroelasticity of profile cascades has not yet been experimentally tested. Therefore the suitability of supposed structural elements, e.g. maintaining for the elastic support of individual profiles and their assembly in cascades, has not yet been verified. A particular feature of the assignment was the possibility of the interferometry application and other optical methods to measure the flow field. For these types of experiments, IT has a unique interferometer of Mach-Zehnder type.

A vacuum aerodynamic tunnel with a small settling chamber was available for the planned experiments. The tunnel scheme in the arrangement for interferometric measurements is in Fig.1.

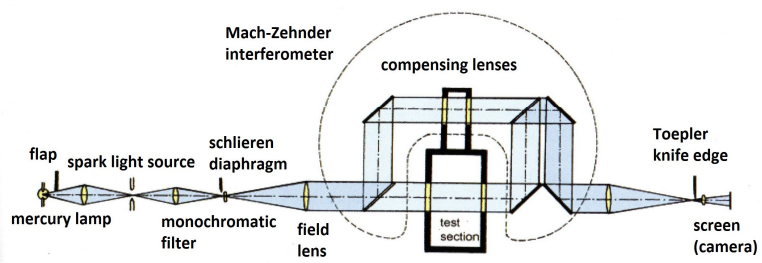

Fig. 1. The scheme of the optical measurements using MachZehnder interferometer.

\section{Requirements for the test section and their design solutions}

It is assumed to use the universal test section not only for experiments on both turbine and compressor cascades, i.e. in a wide range of profile thicknesses and chord curvatures.

\subsection{Test section requirements}

* Construction should be designed with respect to the transparency of the optical windows in the tunnel sidewalls. This condition results from the requirement for the use of a test section for optical measurements using Mach-Zehnder interferometer with optical glasses of a diameter $160 \mathrm{~mm}$.

* Possibility of the rotation of the sliding frame in the range of at least $+/-10^{\circ}$ to allow measurements on cascades at different angles of attack

* It is necessary to use shifting upper and lower walls of the test section in a direction perpendicular to the air flow. By appropriately modifying the length of these adjustable walls, the height and axial position of the inlet walls according to the cascade can be adjusted.

* To allow the profiles two degrees of freedom, pitch separately for each profile and plunge common for all. From the point of view of the assumed practical significance, the possibility of independent plunge of the profiles was not required.

* Use divided profiles to facilitate the assembly of elastic rotation elements, rotary encoder, pressure sensors and their cabling.

* To propose a solution for adjusting the torsional stiffness of the profiles in the flowing air to enable its individual and external changing. This way of setting of torsional stiffness resulted from the need to set the correct geometry of the profile cascade situated in the flowing air before the beginning of the measurement. 
When the profiles in cascade are situated in the nonhomogeneous flow field, we don't know in advance moments acting on their surfaces. Adjusting the correct grid geometry with simultaneous air flow will therefore require individual tuning of the torsional prestressing. Moreover, other velocity value of the airstream will need to re-adjust individual prestressing.

Influence of the turbulence of the input stream is assumed to be accomplished by turbulization grids located in the inlet space of the test section similarly to the research of the self-excited oscillation of the isolated profile NACA 0015 [6].

\subsection{Construction of the test-section}

The existing test section, which was selected for adaptation for aeroelasticity experiments with profile cascades, has the width $80 \mathrm{~mm}$ and the height is up to $420 \mathrm{~mm}$. The width is convenient for the manufacturing of the profile cascades and for the economy of tunnel operation. Less convenient is from the point of view of interferometry. The accuracy of the interferometry depends on the number of interferometric strips and this number linearly increases with the width of the test section and also with velocity of the flow. Therefore interferometry will be in this narrow channel relatively low accurate in the region of low velocities.

The shaping of the inlet (see e.g. [5]) was kept unchanged and is a compromise resulting from the need to allow to change the position of the upper and lower adjustable walls, the turbulence level behind the intake is described in [6].

The mobility and length of upper and lower adjustable walls must be such as to adapt the height of the channel to the position of the outer profiles of the cascade in any position of the profile cascade. The solution is to move the inner part of the duplicate upper and lower walls. The connection to the moving parts of the inlet is realized by a metal strip.

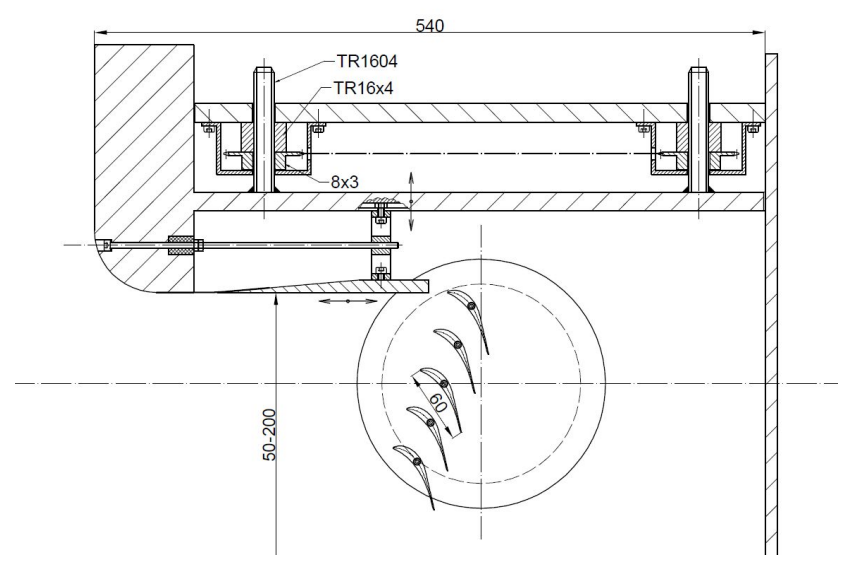

Fig. 2. The scheme of the test section and the controlled adjustable walls.

\subsection{Sliding (supporting) frame}

The sliding frame is guided vertically by parallelogram made from two metal strips. The elasticity of its vertical displacement is ensured by its fitting between two flat adjustable springs.

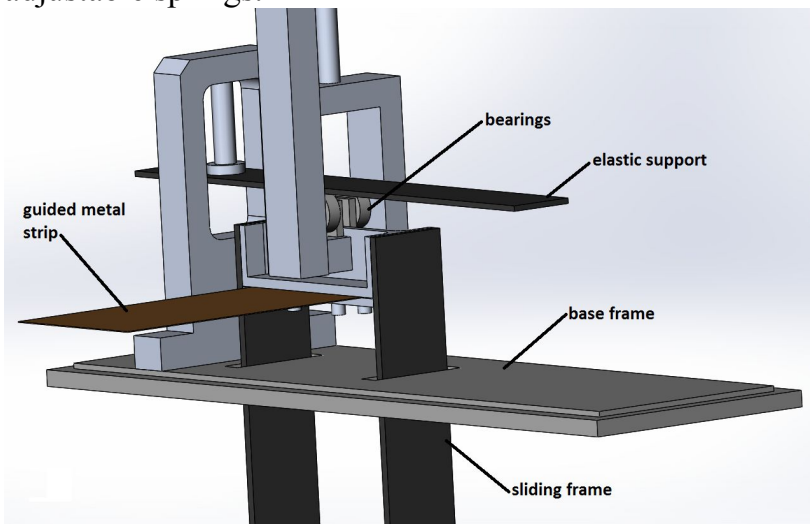

Fig. 3. The scheme of upper part of the sliding support frame.

The frame requires sufficient rigidity against the oscillatory movements perpendicular to the side walls of the test section. During oscillation the frame must not come into contact with optical windows placed in the side walls.

The construction of the sliding frame, including elasticity of its support, is assumed to be taken from the structure of the original tunnel used for aeroelasticity research of the isolated airfoil $[7,8]$. The profile cascade creates separable centre part of the sliding frame.

The dimensions of the supporting frame are based on the requirement to hold up to five profiles in the cascade. For optical measurements, it is necessary to place the profiles in the cascade so, that the measured portion of the flow-field is in optical window of the diameter of $160 \mathrm{~mm}$.

\subsection{Mechanism of rotation of the sliding (supporting) frame}

The solution is to use a thin ball bearing with a diameter of about $300 \mathrm{~mm}$ on one side of the test section (Fig. 4). The inner collar is firmly connected to the construction of the test section and the outer collar is firmly connected to the sliding (support) frame by a rotating frame outside the test section. The rotation of the frame requires the creation of a suitable groove in the upper and lower walls of the tunnel and in the used secondary walls.

\subsection{Construction of the blade cascade}

The elastic support of the profiles situated in the cascade needs to be solved depending on the thickness of the profile, because the construction depends primarily on demand whether an elastic member can be placed inside the profile. However, in the profile cascade, an external correction of the internal elastic member is often required. This member ensures the correct geometry of the cascade during its placing and the air flow setting (the justification is given in section 2.1).

For modelling of real profile cascades, the type TR-B-3 with thin profile and the type SE 1050 as a thick 
one with a large turn of the flow were pre-selected. The cascades were thoroughly measured in IT at the stationary flow mode. Their geometry is depicted in figure 5. For the continuation of previous aeroelastic measurements with isolated profiles, it is also possible to use results obtained formerly with a two-ring 18\% DK18 profile (see [10]).
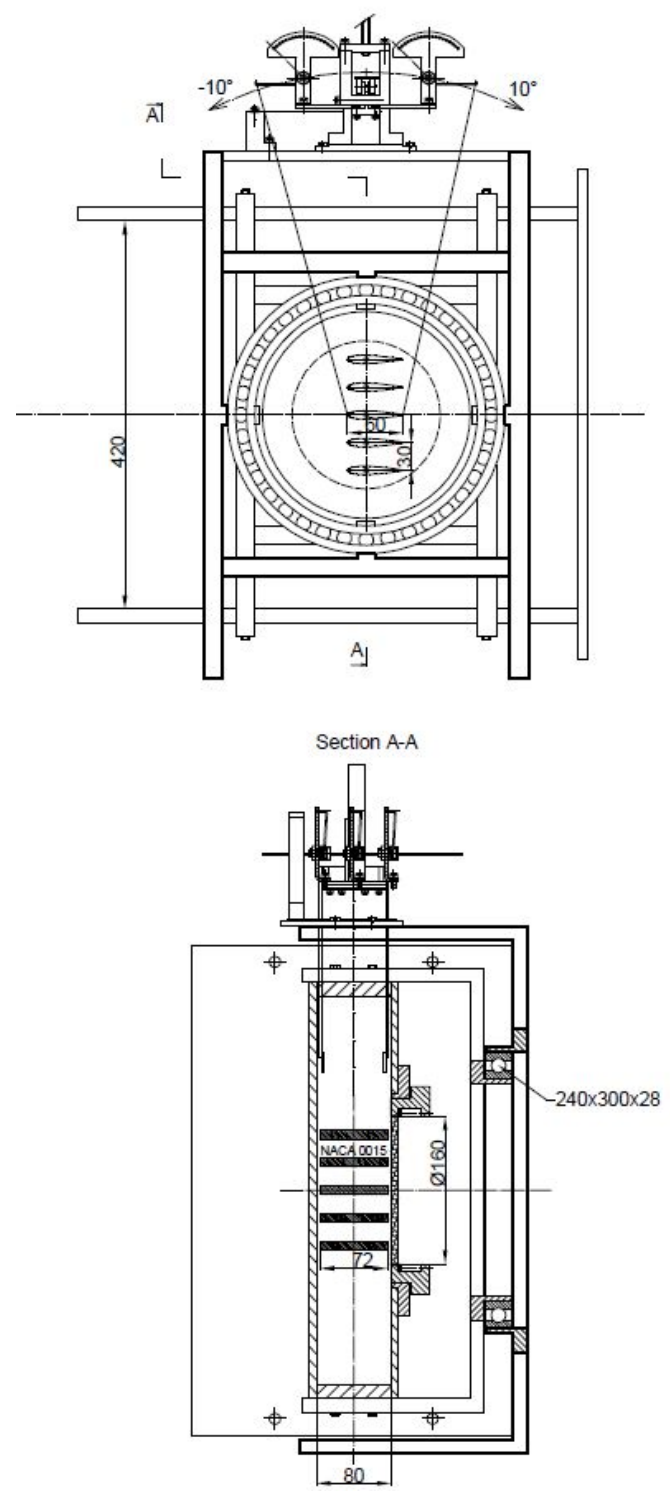

Fig. 4. The scheme of the test section with adjustable angle of attack of the cascade.
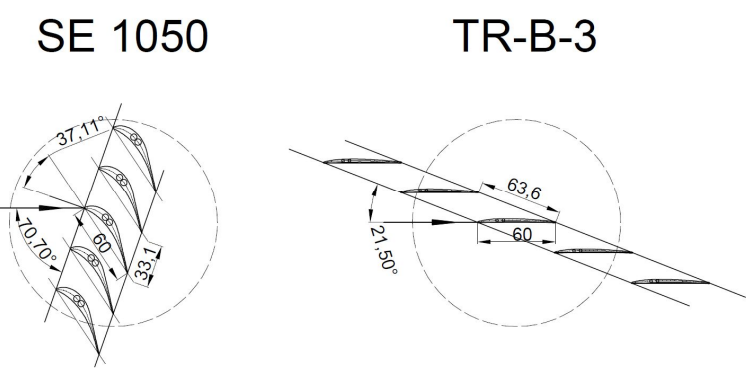

Fig. 5. Drawings of profile cascades. a) SE 1050 , b) TR B-3.

\subsection{Profiles and their internal elastic elements}

Initial experiments on the modified tunnel will be linked to the previous results obtained with the NACA 0015 self-oscillating profile, where the rotation axis was in $1 / 3$ of the chord length. The appropriate position of the axis of rotation for the profiles used in cascades will need to be determined individually (For preliminary experiments we used axis position at $1 / 3$ and $1 / 4$ of chord length.) The design of the profile must allow, in addition to elastic support, installation of the semiconductor pressure sensors, the profile rotation sensor and their connection to the device outside the test section. As a compromise, the chord length of $60 \mathrm{~mm}$ was chosen to allow the standard cascade to be used up to five profiles. This dimension was tested already during the previous aeroelastic experiments and was the smallest dimension that met the requirements of sensors installation. The cross-sectional division of the profile into ten-sections facilitated the placement of the required measuring technique into the profiles. An example of such construction is shown in Fig. 6, 7.

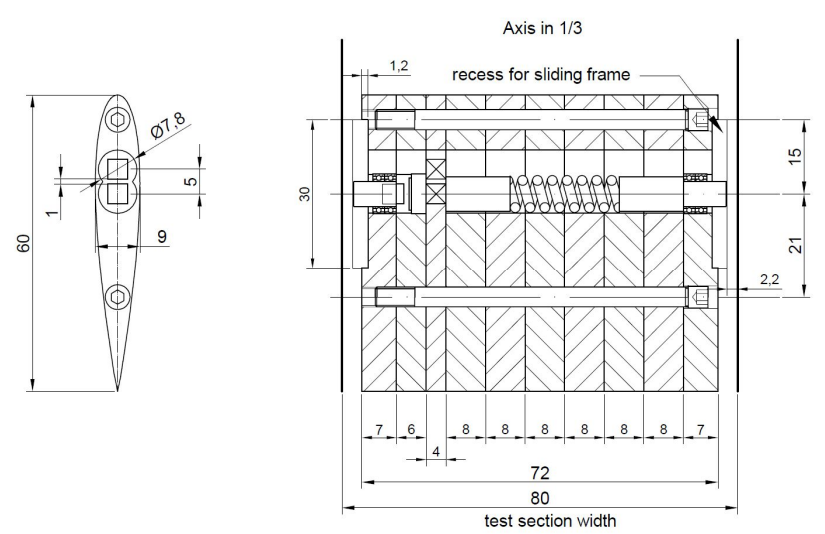

Fig. 6. The scheme of divided profile NACA 0015 with elastic element and rotary encoder.

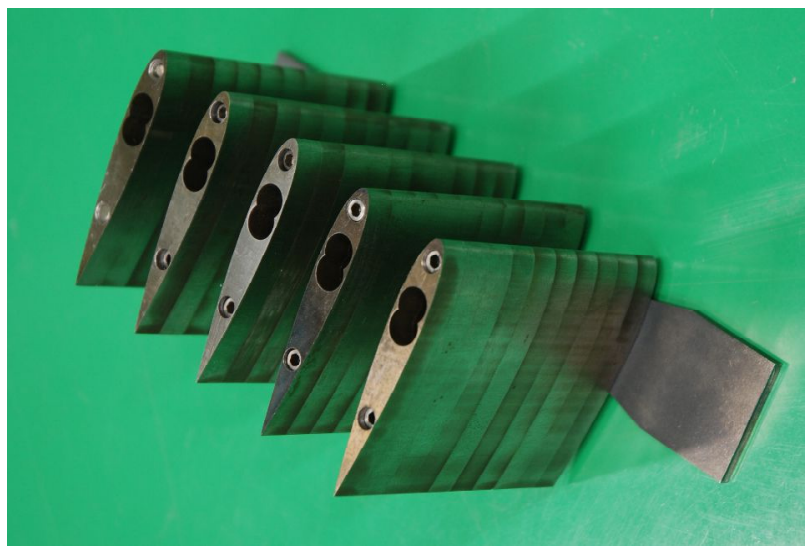

Fig. 7. A photo of blade cascade consisting of five NACA 0015 profiles fixed to support frame.

To estimate the largest torque to be applied by the elastic supports on a $60 \mathrm{~mm}$ chord profile at low subsonic flow velocities, the SE 1050 cascade model was used. For example, with the rotation axis placed at $1 / 4$ of the profile, chord length $60 \mathrm{~mm}$, profile width 80 
$\mathrm{mm}$ and input flow velocity $\mathrm{M}=0.33$, a torque $\mathrm{M}_{K}$ of about $5 \mathrm{Nm}$ was detected.

\section{Torque for SE1050 profile in cascade}

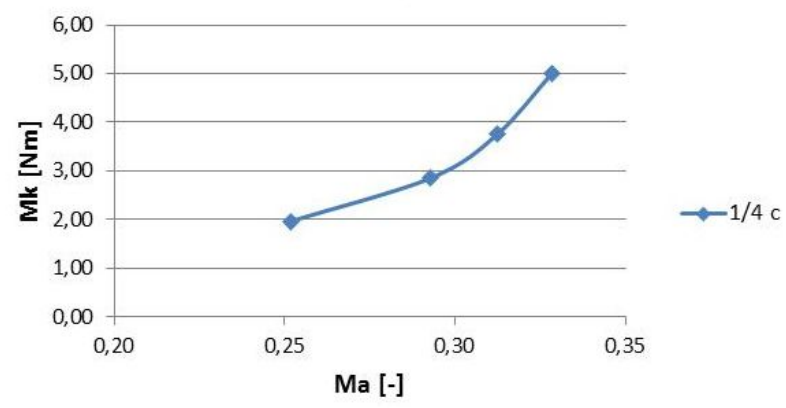

Fig. 8. The torque dependency on inlet Mach number for SE1050 three-blade cascade, measured for centre blade.

\subsection{Design of torsional elastic elements placed outside the test section}

The requirement for the external influence of the support elasticity of the profile rotation results mainly from the need for aeroelastic measurements with thin profiles. In these cases the maximum profile thickness is several millimeters when the length of the profile chord is 60 $\mathrm{mm}$. Then there is no space within the profile for the embedding of the elastic member even when torsion bars are used.

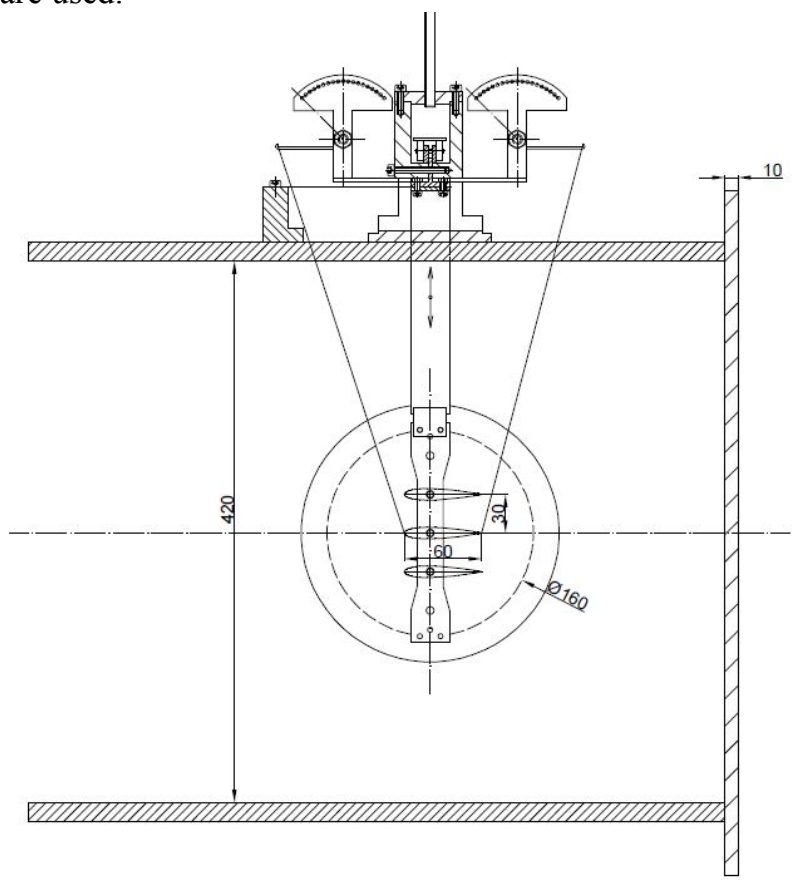

Fig. 9. The schema of the elastic members of the profile rotation, located outside the test section

One possible solution is, for example, fixation of the trailing edges of the profiles on the up and down pulling strings, whose thrust is controlled by springs placed on the sliding frame. Although this system was previously implemented with aeroelastic measurements on the isolated profile, it was not properly tested. The feasibility of the solution is in many cases controversial, especially when measured with non-zero angles attack.

In cases where the profiles in the cascade are significantly shifted in the direction of the axis of the test section, there is an idea to hang up the profiles only on one side and the balancing moment induced by a coil spring thread located on the side of the profile and supported by the side part of the sliding frame.

\subsection{Profile coordinate measuring device}

This device consists of a pointer with a translation and rotation position indicator. The pointer is actually the plexiglass with the profile contour which is to be used to identify the real profile position inside the test section. The coordinates in Cartesian coordinate system and the angle of rotation indicates the reference starting position of the profile in cascade. There is an assumption about the NACA 0015 profile experiments that the starting position of the profile will correspond to final position for the case of self-oscillation in the blade cascade. The mechanical coordinate measuring device is possible to remove when the interferometer is used. Additional information about profile position during oscillation will be acquired from rotary encoder (pitch) and also from accurate laser rangefinder (plunge).

\section{Some results obtained with NACA 0015 self-oscillating airfoil}

This is part of the results obtained with airfoil NACA 0015 during its self-excited vibration. The test section was in the state before it was modified for blade cascades measurements. The drawing of the airfoil is in figure 10 .

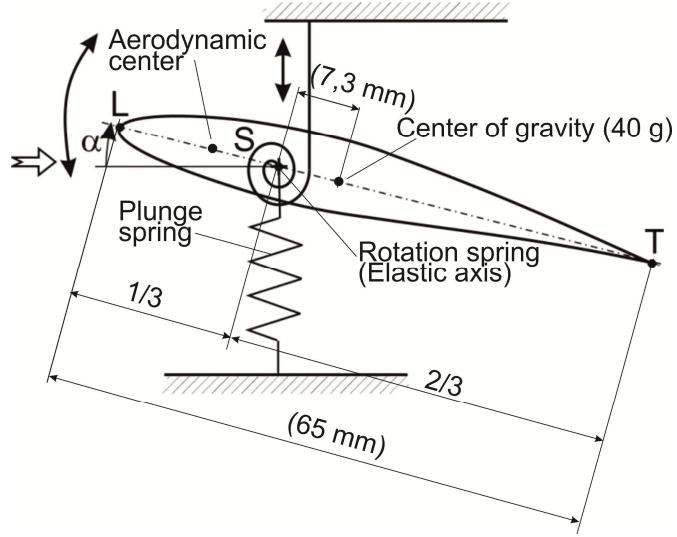

Fig. 10. Experimental setup of isolated profile with two degrees of freedom - pitch $\alpha$ and plunge.

One of the finding valid for this arrangement was the general relationship between the Strouhal number Sh of the self-oscillating profile and the flow Mach number M, see Figure 11 (also see [9]). The characteristic length in Sh was the chord length. 


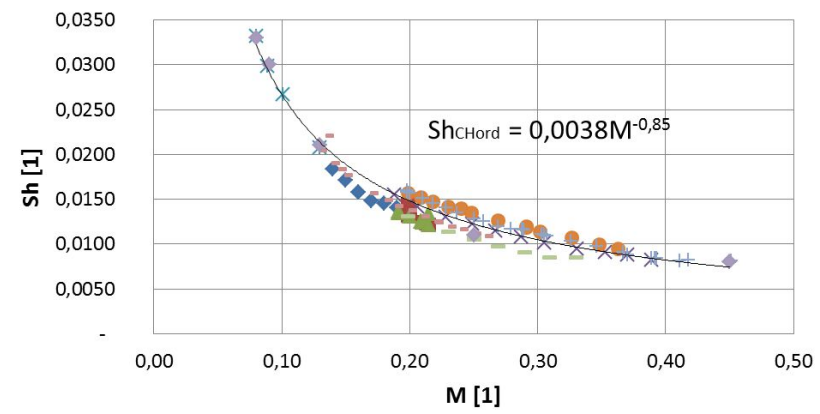

Fig. 11. Relationship between Strouhal and Mach number.

The example of the kinematics of the airfoil during one period of the self-excited vibration is in figure 12 . The red vertical line denotes the trajectory of the centre of rotation during one period. Two black contours depict the upper and lower positions of airfoil parallel to the airstream direction. The profile pitch angle reached the value up to $+/-45^{\circ}$. The plunge peak-to-peak value was up to $10 \%$ of the chord length.

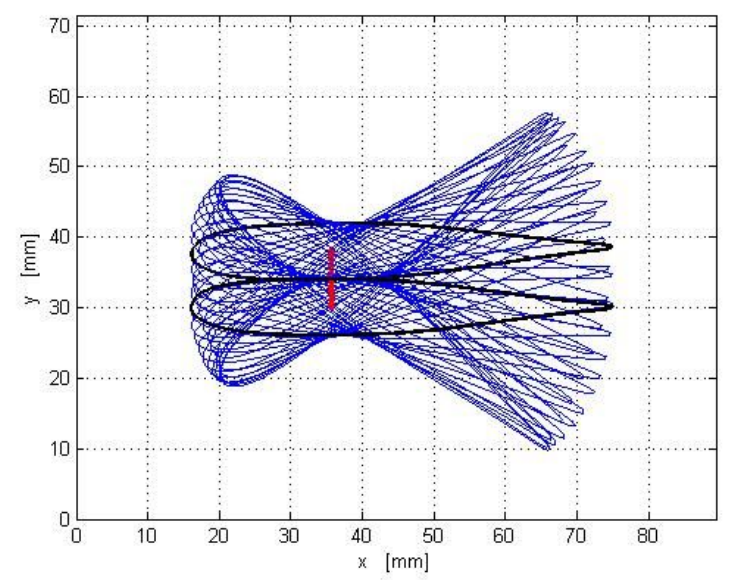

Fig. 12. The kinematics of the airfoil corresponding to the interferograms $2663-05, M=0,45$.

The last figures are presented here to show the interferograms of the flow field around the airfoil during one period of self-excited oscillation. The velocity of the flow was $\mathrm{M}=0.45$, a scanning rate was 1000 fps. Interferometry indicates the density deviations and consequently it is possible to evaluate the velocity and pressure field assuming isentropic changes in the flow field. The figures show the total flow separation which is periodical during self-excited oscillation.

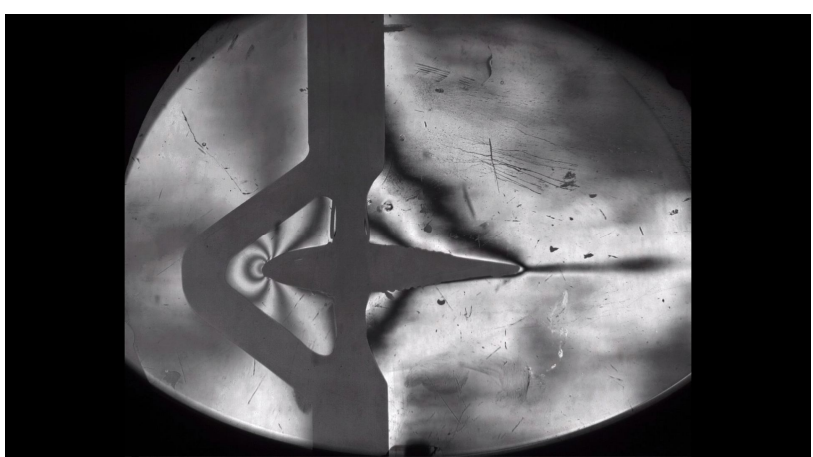

Fig. 13a. Interferogram, airfoil at bottom dead centre.

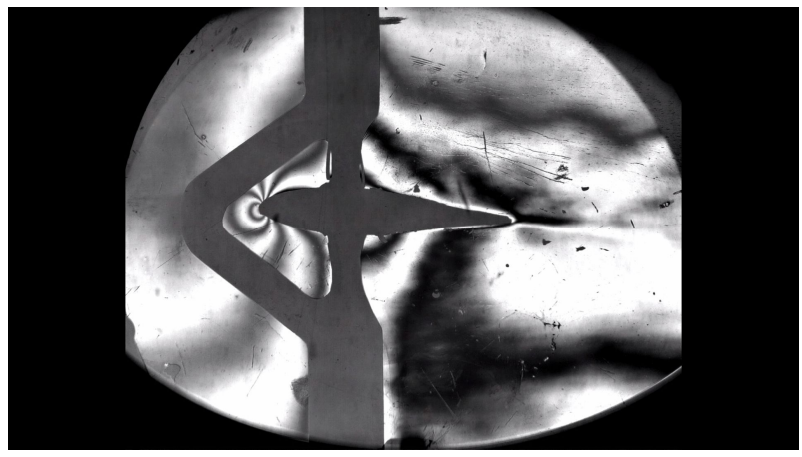

Fig. 13b. Interferogram, airfoil at top dead centre.

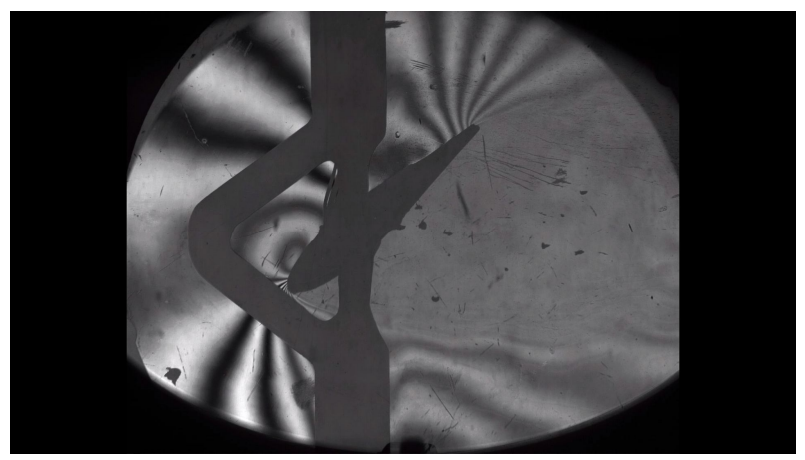

Fig. 13c. Interferogram, airfoil at maximal negative inclination.

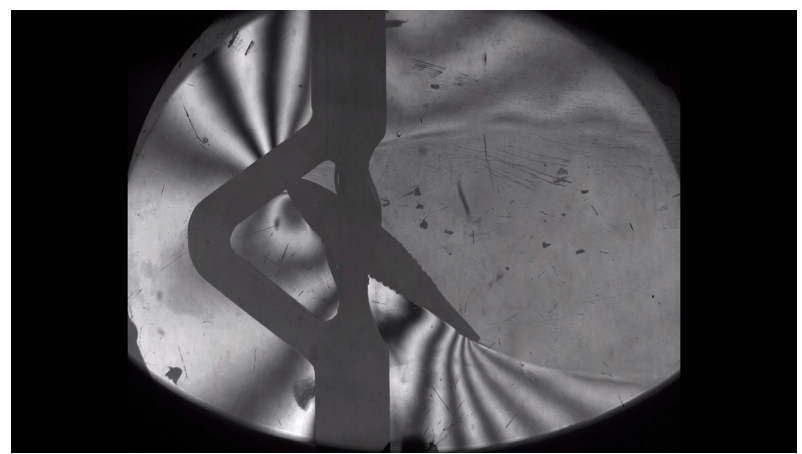

Fig. 13d. Interferogram, airfoil at maximal positive inclination. 


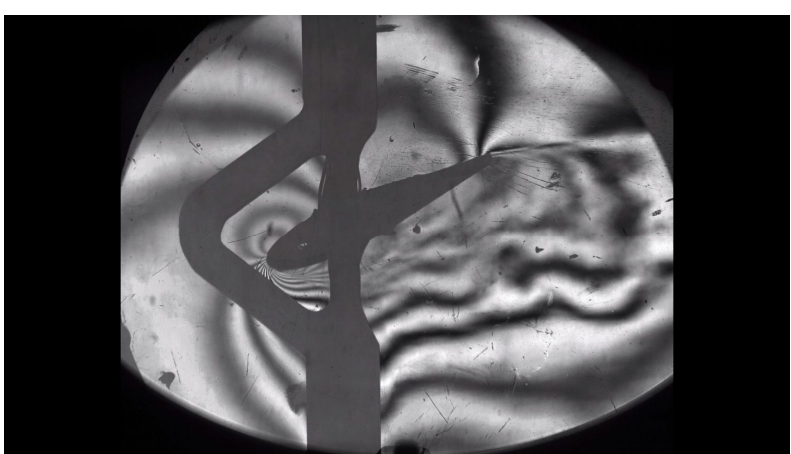

Fig. 13e. Interferogram, separated flow at lower airfoil side.

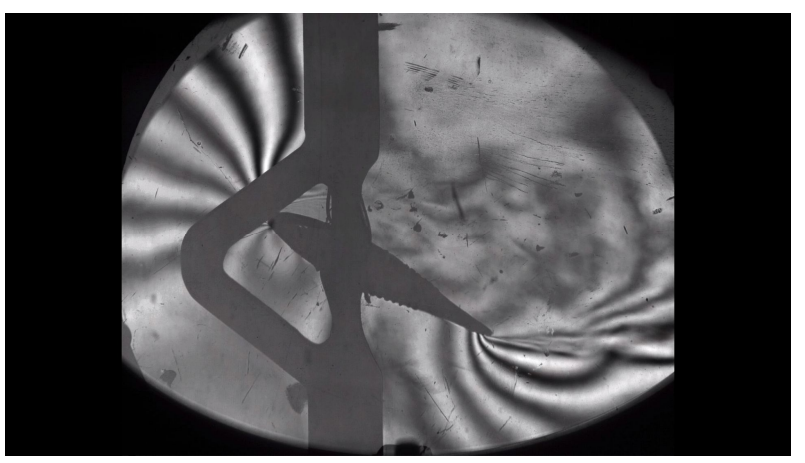

Fig. 13f. Interferogram, separated flow at upper airfoil side.

\section{Conclusion}

The modifications of the classic test section of the small aerodynamic tunnel have been designed and partially implemented. This is an extension of the modifications, designed and tested in the IT during the research of selfexcited oscillation of isolated profiles; as a two-circle $18 \%$ profile and airfoil NACA 0015 . Modifications of the tunnel construction are directed to the possibility of studying aeroelasticity of the self-excited oscillation of symmetrical and nonsymmetrical profiles located in the blade cascade both of the compressor and the turbine types.

This work was supported by the research project of the Institute of Thermomechanics "Experimental investigation of selfexcited oscillations of a blade cascade".

\section{References}

1. Francsson T.H. (ed.), Unsteady aerodynamics, aeroacoustics and aeroelasticity of turbomachines. Springer, (1998)

2. Hall K.C., Kielb R.E., Thomas J.P. (eds), Unsteady aerodynamics, aeroacoustics and aeroelasticity of turbomachines. Springer, Dordrech, (2006)

3. Stenfelt G., Ringertz U., The Aeronautical Journal, 119, No.1222, (2015)

4. Razak N.A., Dndrianne T., Dimidtriadis G., AIAA Journal, 49, 10, (2011)

5. Fuh-Min Fang Chen, Hong Y.T., Journal of Wind Engineering and Industrial Aerodynamics, 89, 3-4, (2001)
6. Šidlof P., Antoš P., Šimurda D., Štěpán M., Dančová P., EPJ Web of Conferences 143, (2017)

7. Šidlof P., Vlček V., Štěpán M, Journal of Fluids and Structures, 67, (2016)

8. Vlček V., Štěpán M. Zolotarev. I., Kozánek. J., Applied Mechanics and Materials, 821, (2016)

9. Vlček V., Zolotarev. I., Kozánek. J., Šidlof P., Štěpán M., International Colloquium Dynamesi (2017)

10. Horáček J., Sváček P., Vlček V., Feistauer M., IFASD 2009 Proceedings. Seattle Washington: Azimuth Corporation, (2009) 